Revista electrónica de AnestesiaR

PROTOCOLO

Abril 2019

\title{
Protocolo de prevención de la hipotermia perioperatoria
}

Miró Murillo M, Recio Pérez J, Salinero Fernández P, Paz Pacheco EM.

HU Torrejón, Madrid.

\section{Resumen}

La hipotermia perioperatoria es una complicación muy frecuente en nuestro medio que compromete la seguridad de nuestros pacientes quirúrgicos aumentando la morbimortalidad perioperatoria. La hipotermia también aumenta de manera considerable los costes sanitarios por el aumento de las complicaciones y la estancia hospitalaria. Recientemente se ha publicado la guía de práctica clínica de hipotermia perioperatoria no intencionada de la SEDAR. En esta guía se presenta la evidencia científica actual sobre la hipotermia perioperatoria y las recomendaciones sobre su manejo.

Basado en esta guía, presentamos el protocolo de prevención de la hipotermia perioperatoria de nuestro centro con una estrategia basada en la monitorización de la temperatura central y en 3 medidas que han demostrado disminuir las complicaciones perioperatorias y ser coste-efectivas: el uso de mantas de aire convectivo, el calentamiento de los fluidos intravenosos y de irrigación, y el calentamiento activo previo a la cirugía.

\section{Introducción}

La hipotermia perioperatoria es una complicación muy frecuente en nuestro medio que compromete la seguridad de nuestros pacientes quirúrgicos aumentando la morbimortalidad perioperatoria. La hipotermia también aumenta de manera considerable los costes sanitarios por el aumento de las complicaciones y la estancia hospitalaria.

Recientemente se ha publicado la guía de práctica clínica de hipotermia perioperatoria no intencionada de la SEDAR. En esta guía se presenta la evidencia científica actual sobre la hipotermia perioperatoria y las recomendaciones sobre su manejo.

Basado en esta guía, presentamos el protocolo de prevención de la hipotermia perioperatoria de nuestro centro con una estrategia basada en la monitorización de la temperatura central $\mathrm{y}$ en 3 medidas que han demostrado disminuir las complicaciones perioperatorias y ser coste-efectivas: el uso de mantas de aire convectivo, el calentamiento de los fluidos intravenosos y de irrigación, y el calentamiento activo previo a la cirugía.

\section{Objeto}

El objeto del protocolo se basa en definir la hipotermia perioperatoria, sus características y las complicaciones derivadas de ella. Determinar cuáles son los factores de riesgo implicados y la estrategia para su prevención y manejo.
Alcance
Aplica al bloque quirúrgico (CMA, quirófanos y URPA) y UVI. 


\section{Términos y definiciones}

- ASA: Escala de riesgo anestésico de la American Asociation of Anesthesiolgy

- Compartimento central: parte del organismo que genera calor (depende la actividad metabólica de los órganos internos como el cerebro y los órganos de las cavidades abdominal y torácica).

- Compartimento periférico: parte del organismo que regula la pérdida de calor.

- Conducción: fenómeno consistente en la propagación de calor entre 2 cuerpos o partes de un mismo cuerpo a diferente temperatura debido a la agitación térmica de las moléculas, no existiendo un desplazamiento real de éstas.

- Convección: transporte de calor por medio del movimiento de un fluido (por ejemplo, el aire).

- Evaporación: pérdida de calor por paso de líquido a gas.

- Hipotermia: descenso de la temperatura del compartimento central por debajo de $36^{\circ} \mathrm{C}$.

- Líquidos de irrigación: aquellos líquidos que se emplean durante la cirugía para distender una cavidad (vejiga, útero, cavidades articulares) con el fin de mejorar las condiciones quirúrgicas.

- Método activo de calentamiento: cualquier método que aumenta la temperatura corporal total mediante calentamiento bien externo, bien interno.

- Precalentamiento: aplicación de calor antes de la anestesia y/o cirugía con el propósito de aumentar la temperatura corporal total.

- Radiación: transmisión de calor entre 2 cuerpos los cuales, en un instante dado, tienen temperaturas distintas, sin que entre ellos exista contacto.

- Sistema de aire forzado o aire caliente convectivo: sistema de calentamiento que emplea aire caliente para transferir calor al organismo.

\section{Estrategia de manejo}

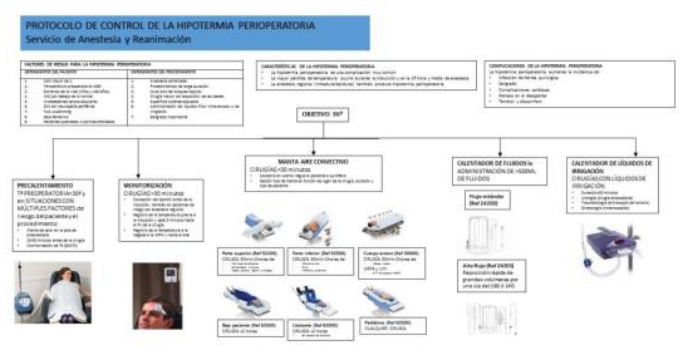

Clic para ver ampliado

\section{Descripción del Protocolo}

\section{HIPOTERMIA \\ PERIOPERATORIA: FISIOLOGÍA Y CONSECUENCIAS}

La hipotermia es una complicación muy frecuente en el periodo perioperatorio, hay estudios que demuestran una incidencia del 56,29\% (1).

\section{Los mecanismos fisiológicos que} favorecen la hipotermia perioperatoria son: la termorregulación inefectiva y la vasodilatación, la redistribución del calor desde el compartimento central al compartimento periférico, la exposición del paciente sin cubrir a la baja temperatura de quirófano y la administración de líquidos fríos por vía intravenosa $\mathrm{o}$ de irrigación. $\mathrm{El}$ mecanismo fundamental de pérdida de calor es la irradiación (figura 1). La cinética de la pérdida de calor sigue tres fases como se ve en la figura 2, siendo la pérdida más rápida en la $1^{\mathrm{a}}$ hora desde la inducción anestésica (2). 


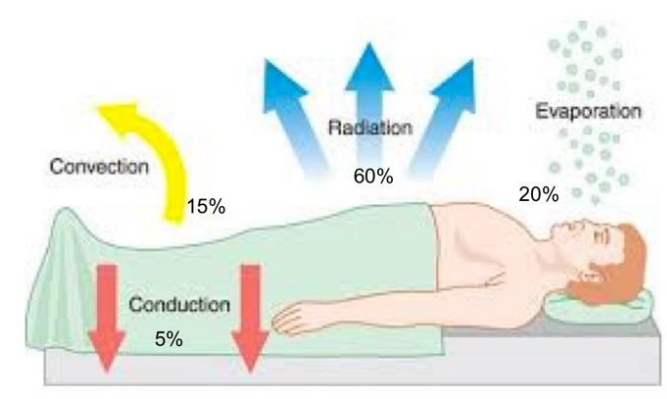

Figura 1. Mecanismos de pérdida de temperatura. Tomado de Sessler DI. Perioperative heat balance. Anesthesiology. 2000 Feb;92(2):578-96

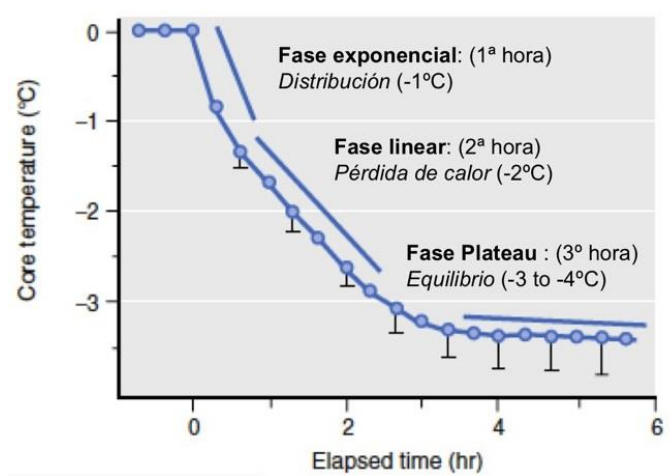

Figura 2. Cinética de la pérdida de temperatura. Tomado de Sessler DI. Perioperative heat balance. Anesthesiology. 2000 Feb;92(2):57896

La hipotermia perioperatoria aumenta la morbimortalidad y los costes sanitarios en el periodo perioperatorio. Aumenta la incidencia de infección de herida quirúrgica [RR 3.25 CI I5\% $_{\%}$ (1.357.84)], la coagulopatía [RR 4.49 $\mathrm{CI}_{95 \%}$ (1.00-20.16)], las complicaciones cardiacas [RR $1.33 \mathrm{CI}_{95 \%}$ (1.06-1.66)], retrasa el despertar del paciente, produce temblores y disconfort en el paciente y aumenta la mortalidad del paciente politraumatizado (3).

El mantenimiento de la normotermia durante el periodo perioperatorio reduce los costes asociados al procedimiento al disminuir las complicaciones (fundamentalmente la infección de herida quirúrgica) y la estancia hospitalaria. Según un metaanálisis realizado en EEUU este ahorro estaría estimado entre los 2.500 7.000 USD\$ por procedimiento (4).

Según la guía de práctica clínica de la SEDAR, en las personas adultas que van a ser sometidas a cirugía que requieran anestesia general o regional, se recomienda el uso de algún método activo de prevención de la hipotermia durante el perioperatorio (recomendación fuerte) (5).

\section{FACTORES DE RIESGO PARA SUFRIR HIPOTERMIA PERIOPERATORIA}

En la consulta de preanestesia se debe evaluar la presencia de factores de riesgo de hipotermia perioperatoria (6). Estos factores de riesgo son (5):

\begin{tabular}{|c|c|c|}
\hline $\begin{array}{l}\text { DEPENDIENTES DEL } \\
\text { PACIENTE }\end{array}$ & $\begin{array}{l}\text { DEPENDIENTES DEL } \\
\text { PROCEDIMIENTO }\end{array}$ & AMBIENTALES \\
\hline $\begin{array}{l}\text { 1. ASA mayor de } 1 . \\
\text { 2. Temperatura } \\
\text { preoperatoria }>36^{\circ} \\
\text { 3. Extremos de la vida } \\
\text { (niños y }>60 \text { años) } \\
\text { 4. IMC por debajo de lo } \\
\text { normal } \\
\text { 5. Antecedentes } \\
\text { cardiovasculares } \\
\text { 6. DM con neuropatia } \\
\text { periférica } \\
\text { 7. TAS < } 140 \mathrm{mmHg} \\
\text { 8. Sexo femenino } \\
\text { 9. Pacientes quemados } \\
\text { politraumatizados }\end{array}$ & $\begin{array}{l}\text { 10. Anestesia combinada } \\
\text { 11. Procedimientos de larga } \\
\text { duración } \\
\text { 12. Nivel alto del bloqueo } \\
\text { espinal } \\
\text { 13. Cirugia mayor con } \\
\text { exposición de cavidades } \\
\text { 14. Superficie cutánea } \\
\text { expuesta } \\
\text { 15. Administración de liquidos } \\
\text { frios intravenosos o de } \\
\text { irrigación } \\
\text { 16. Sangrado importante }\end{array}$ & $\begin{array}{l}\text { 17. Baja } \\
\text { temperatura de } \\
\text { quirófano }\end{array}$ \\
\hline
\end{tabular}

\section{ESTRATEGIA DE PREVENCIÓN DE LA HIPOTERMIA PERIOPERATORIA}

La estrategia de prevención de la hipotermia perioperatoria en nuestro centro consta de $\mathbf{4}$ pilares:

1. MONITORIZACIÓN

2. USO DE MANTAS DE AIRE CONVECTIVO

3. CALENTAMIENTO DE LÍQUIDOS INTRAVENOSOS Y DE IRRIGACIÓN

4. PRECALENTAMIENTO

\section{I.- MONITORIZACIÓN}

La ASA (American Society of Anesthesiology) publica en 1986 los estándares de monitorización para 
la anestesia. Incluye que se debe monitorizar la temperatura en todo paciente en el que se prevea que se van a producir cambios significativos de la misma. La SEDAR amplió esta recomendación a todo procedimiento con una anestesia general con una duración más de 30 minutos o cirugía de más de 1 hora (5). La incidencia de hipotermia perioperatoria en pacientes con anestesia neuroaxial es muy alta (7) por lo que se considera que en pacientes con este tipo de anestesia también es fundamental monitorizar la temperatura central (5). A pesar de las recomendaciones la monitorización sigue sin ser un estándar de monitorización $(1,8,9)$.

Las indicaciones sobre la monitorización de la temperatura en el periodo perioperatorio son (6): $\quad$ se debe monitorizar siempre la temperatura central siendo el objetivo mantener una $T^{a}>36^{\circ} \mathrm{C} \quad \mathrm{Se}$ debe monitorizar y registrar la temperatura en todo procedimiento que dure más de 30 minutos antes de pasar a quirófano, durante el periodo intraoperatorio y en la URPA hasta el alta. No se debe comenzar ninguna inducción anestésica con un paciente con una $\mathrm{T}^{\mathrm{a}}<36^{\circ} \mathrm{C}$ (exceptuando la cirugía urgente/emergente). No se debe dar de alta de la URPA a ningún paciente con una $\mathrm{T}^{\mathrm{a}}<36^{\circ} \mathrm{C}$.

El monitor spot-on (3M®), a través de la tecnología ZHF (zero heat flux), proporciona una estimación de la temperatura central midiendo la temperatura de 1-2 cm bajo la piel de la sien a través de un túnel isotérmico que se genera bajo el sensor. Proporciona una medida no invasiva, rápida $\mathrm{y}$ continua de la temperatura central (10). Este monitor se ha validado con el catéter de arteria pulmonar $(11,12)$, con el termómetro esofágico $(13,14)$, con el termómetro nasofaríngeo y sublingual (15). También se puede utilizar en pacientes a los que se realiza craneotomía (16). Este monitor está conectado con el monitor de anestesia y se registra de manera automática en la gráfica de anestesia ICCA (Philips ${ }^{\circledR}$ ).

\section{II.- USO DE MANTAS DE AIRE CONVECTIVO}

Las mantas de aire convectivo han demostrado disminuir las complicaciones asociadas a la hipotermia perioperatoria (17) y han demostrado ser una medida costeefectiva en estudios económicos (6).

Funcionan reemplazando el aire frío en contacto con el paciente y transfiriendo el calor desde la superficie al compartimento central. Sirven tanto para prevenir la hipotermia como parar tratarla una vez instaurada. Son fáciles de utilizar y seguras.

Las indicaciones para el uso de las manta de aire convectivo son: Se deben utilizar en toda cirugía con una duración de más de 30 minutos o en cualquier paciente con factores de riesgo independientemente de la duración de la cirugía (6).La manta debe colocarse y conectarse cuanto antes una vez el paciente está en la mesa de quirófano (durante la colocación, monitorización y realización de la anestesia neuroaxial) ya que la pérdida de calor más rápida se produce en esa fase y en la inducción anestésica. El tipo de manta debe ajustarse al lugar de la cirugía y la duración de la misma según el algoritmo del protocolo. La manta bajo paciente es la más efectiva a la hora de mantener la normotermia y se debe utilizar siempre que se pueda en cirugías de $>2$ horas de duración. Sin embargo, son las más complicadas de utilizar y hay que seguir una serie de recomendaciones a la hora de su colocación (ANEXO 1). En ningún caso se utilizará el motor de aire caliente sin 
la manta correspondiente (goosing) por el riesgo de quemaduras e incendio.

\section{III.- CALENTAMIENTO DE FLUIDOS INTRAVENOSOS Y DE IRRIGACIÓN}

El calentamiento de los fluidos de administración intravenosa y/o de irrigación ha demostrado ser eficaz en el mantenimiento de la normotermia perioperatoria (18) y ser una medida coste-efectiva (6). Estos sistemas previenen la hipotermia, pero, por sí solos, no son capaces de tratarla. Por esta razón siempre deben utilizarse como complemento a otros sistemas.

Las indicaciones para el uso de calentadores de fluidos son: Se deben utilizar en cirugías en las que se vayan a administrar $>500 \mathrm{ml}$ de fluidoterapia intravenosa (6).En cirugías en las que se prevea necesidad de gran cantidad de fluidos se utilizará un sistema de alto flujo conectado a una vía de calibre 16G-14G.Se utilizará calentadores de líquido de irrigación en las cirugías en las que se utiliza una cantidad significativa de líquidos de irrigación y/o de larga duración: cirugía endourológica, histeroscopias y artroscopia de hombro.

\section{IV.- PRECALENTAMIENTO}

Las estrategias de calentamiento activo previas a la cirugía reducen los gradientes de temperatura entre el compartimento central y periférico, minimizan la redistribución de calor y por lo tanto previenen la hipotermia (19). También han demostrado disminuir la infección de herida quirúrgica (10 casos menos por cada 100 tratados) (20). Sin embargo, no está claro cuál es la duración adecuada de estas estrategias ni su superioridad frente al calentamiento activo precoz (alrededor de la inducción anestésica) (5).

Las recomendaciones para el uso de estrategias de precalentamiento activo previa a la cirugía son: Se deben realizar siempre en pacientes cuya temperatura preoperatoria sea $<36^{\circ} \mathrm{C}$.Se deben realizar individualizando en situaciones donde se acumulen múltiples factores de riesgo para la hipotermia tanto del paciente como del procedimiento. El procedimiento a seguir será: a) se realizará en la sala de preoperatorio bajo la supervisión de enfermería, b) se utilizarán mantas de aire convectivo que cubran la mayor parte posible de la superficie corporal, c) se monitorizará en todo momento la temperatura con el Spot-On para mantener un rango entre $36-37^{\circ} \mathrm{C}$, d) el precalentamiento se realizará durante un tiempo no inferior a 10 minutos, e) la interrupción del precalentamiento activo para el traslado a quirófano no debe superar los 10 minutos

\section{Monitorización del seguimiento del Protocolo}

La monitorización del seguimiento del protocolo de prevención de la hipotermia perioperatoria se hará siguiendo un ciclo de auditoría clínica (Figura 3).

- Estándar: se toma como estándar de práctica clínica el protocolo presentado.

- Auditoría: 1 semana al año se realizará una auditoría clínica para estudiar el grado de cumplimiento del protocolo

- Análisis de datos: se analizarán los datos de la auditoría y se identificarán fallos y áreas de mejora.

- Implementación de las mejoras: se implementarán las mejoras 
propuestas (formación, cambios en el protocolo...).

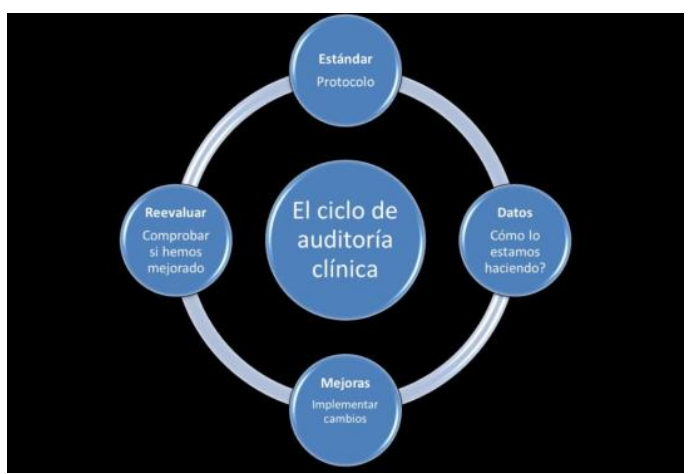

Figura 3. El ciclo de auditoría clínica

La auditoría la llevará a cabo el anestesiólogo y la enfermería de URPA recogiendo los datos de todas las cirugías de una semana. Los datos que se recogerán son:

- Fecha y hora de salida de quirófano

- Temperatura en URPA (Variable principal)

- Especialidad quirúrgica

- Número de historia clínica

- Factores del paciente: edad, ASA

- Factores del procedimiento: tipo de anestesia, duración, cantidad total de fluidos.

- Estrategia de prevención de la hipotermia: monitorización de la temperatura, uso de manta de calor, uso de calentador de fluidos.

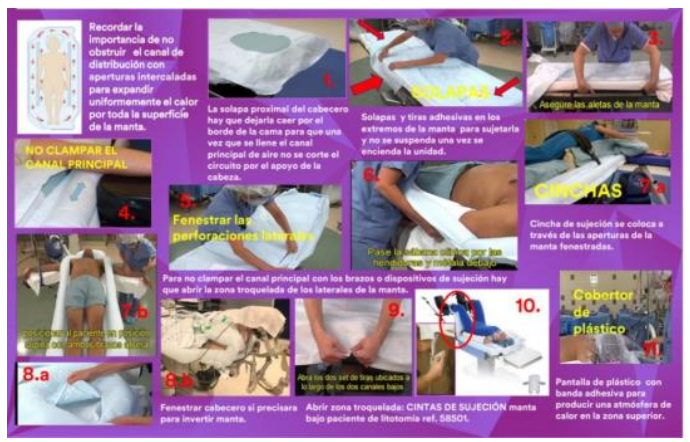

Anexo 1

\section{Bibliografía}

1. Castillo Monzón CG, Candia Arana CA, Marroquín Valz HA, Aguilar Rodríguez F, Benavides Mejía JJ, Alvarez Gómez JA. Temperature management during the perioperative period and frequency of inadvertent hypothermia in a general hospital. Colomb J Anesthesiol. 2013 Apr 1;41(2):97103. (PDF)

2. Sessler DI. Perioperative heat balance. Anesthesiology. $2000 \quad$ Feb;92(2):578-96. (PubMed) (HTML)

3. Torossian A, Bräuer A, Höcker J, Bein B, Wulf H, Horn E-P. Preventing inadvertent perioperative hypothermia. Dtsch Arzteblatt Int. 2015 Mar 6;112(10):166-72. (Mㅡ) (마)

4. Mahoney CB, Odom J. Maintaining intraoperative normothermia: a meta-analysis of outcomes with costs. AANA J. 1999 Apr;67(2):155-63. (PubMed)

5. Calvo Vecino JM, Casans Francés R, Ripollés Melchor J, Marín Zaldívar C, Gómez Ríos MA, Pérez Ferrer A, et al. Guía de práctica clínica de hipotermia perioperatoria no intencionada. Rev Esp Anestesiol Reanim. 2018 Dec 1;65(10):564-88. (․ML)

6. National Collaborating Centre for Nursing and Supportive Care (UK). The Management of Inadvertent Perioperative Hypothermia in Adults [Internet]. London: Royal College of Nursing (UK); 2008 [cited 2019 Mar 29]. (National Institute for Health and Clinical Excellence: Guidance). Available from: http://www.ncbi.nlm.nih.gov/books/NBK 53797/

7. Arkiliç CF, Akça O, Taguchi A, Sessler DI, Kurz A. Temperature monitoring and management during neuraxial anesthesia: an observational study. Anesth Analg. 2000 Sep;91(3):662-6. (ubMed)

8. Torossian A, TEMMP (Thermoregulation in Europe Monitoring and Managing Patient Temperature) Study Group. Survey on intraoperative temperature management in Europe. Eur J Anaesthesiol. 2007 Aug;24(8):668-75. (ubMed)

9. Brogly N, Alsina E, de Celis I, Huercio I, Dominguez A, Gilsanz F. Perioperative temperature control: Survey on current practices. Rev Esp Anestesiol Reanim. 2016 Apr;63(4):207-11. (PubMed) 
10. Schell-Chaple HM, Liu KD, Matthay MA, Puntillo KA. Rectal and Bladder Temperatures vs Forehead Core Temperatures Measured With SpotOn Monitoring System. Am J Crit Care Off Publ Am Assoc Crit-Care Nurses. 2018 Jan;27(1):43-50. ( PubMed) (

11. Mäkinen M-T, Pesonen A, Jousela I, Päivärinta $J$, Poikajärvi S, Albäck $A$, et al. Novel Zero-Heat-Flux Deep Body Temperature Measurement in Lower Extremity Vascular and Cardiac Surgery. J Cardiothorac Vasc Anesth. 2016 Aug;30(4):973-8. (ubMed)

12. Eshraghi Y, Nasr V, Parra-Sanchez I, Van Duren A, Botham M, Santoscoy T, et al. An evaluation of a zero-heat-flux cutaneous thermometer in cardiac surgical patients. Anesth Analg. 2014 Sep;119(3):543-9. (PubMed)

13. Kollmann Camaiora A, Brogly N, Alsina E, de Celis I, Huercio I, Gilsanz F. Validation of the Zero-Heat-Flux thermometer (SpotOn $\left.{ }^{\circledR}\right)$ in major gynecological surgery to monitor intraoperative core temperature: a comparative study with esophageal core temperature. Minerva Anestesiol. 2018 Jun 26 (ubMed)

14. Boisson M, Alaux A, Kerforne T, Mimoz O, Debaene B, Dahyot-Fizelier $\mathrm{C}$, et al. Intraoperative cutaneous temperature monitoring with zero-heat-flux technique (3M SpotOn) in comparison with oesophageal and arterial temperature: A prospective observational study. Eur J Anaesthesiol. 2018;35(11):825-30. (PubMed)

15. Iden T, Horn E-P, Bein B, Böhm R, Beese J, Höcker J. Intraoperative temperature monitoring with zero heat flux technology (3M SpotOn sensor) in comparison with sublingual and nasopharyngeal temperature: An observational study. Eur J Anaesthesiol. 2015 Jun;32(6):38791. (PubMed)

16. Pesonen E, Silvasti-Lundell M, Niemi TT, Kivisaari R, Hernesniemi J, Mäkinen M-T. The focus of temperature monitoring with zero-heat- flux technology (3M Bair-Hugger): a clinical study with patients undergoing craniotomy. J Clin Monit Comput. 2018 Nov 22. (ubMed)

17. Madrid E, Urrútia G, Roqué i Figuls $M$, Pardo-Hernandez H, Campos JM, Paniagua P, et al. Active body surface warming systems for preventing complications caused by inadvertent perioperative hypothermia in adults. Cochrane Database Syst Rev. 2016 Apr 21;4:CD009016. (PubMed) (․MTML)

18. Campbell G, Alderson P, Smith AF, Warttig $\mathrm{S}$. Warming of intravenous and irrigation fluids for preventing inadvertent perioperative hypothermia. Cochrane Database Syst Rev. 2015 Apr 13;(4):CD009891. (PubMed) (HTML)

19. Grote R, Wetz AJ, Bräuer A, Menzel M. [Prewarming according to the AWMF S3 guidelines on preventing inadvertant perioperative hypothermia 2014 : Retrospective analysis of 7786 patients]. Anaesthesist. 2018 Jan;67(1):27-33. (PubMed)

20. Melling AC, Ali B, Scott EM, Leaper DJ. Effects of preoperative warming on the incidence of wound infection after clean surgery: a randomised controlled trial. Lancet Lond Engl. 2001 Sep 15;358(9285):876-80. (PubMed)

Correspondencia al autor

Miguel Miró Murillo mmiromurillo@outlook.com

Jefe de servicio de anestesia, reanimación y tratamiento del dolor.

HU Torrejón, Madrid.

Aceptado para el blog en abril de 2019. 